\title{
MALARIA VACCINATION AND REBOUND MALARIA
}

\section{Alassane Dicko ${ }^{1}$ Brian Greenwood ${ }^{2}$}

${ }^{1}$ Malaria Research and Training Centre, University of Bamako, Bamako, Mali.

${ }^{2}$ Faculty of Infectious and Tropical Diseases, London School of Hygiene \& Tropical Medicine, London, UK.

Corresponding author: Prof. Brian Greenwood, Faculty of Infectious and Tropical Diseases, London School of Hygiene \& Tropical Medicine, Keppel St., London WC1E 7HT, UK. (brian.greenwoood@lshtm.ac.uk) 
A large phase 3 trial of the RTS,S/AS01 malaria vaccine showed moderate efficacy against severe and uncomplicated malaria ${ }^{1}$ but also raised a number of safety concerns. ${ }^{1,2}$ For these reasons, WHO recommended three, large scale pilot implementation studies ${ }^{3}$ which are now underway. ${ }^{4}$ Infants and children recruited to the phase 3 trial were followed for an initial period of three or four years respectively and it is uncertain whether the efficacy observed in the trial would be sustained. Two recent studies provide further information on the longer term efficacy and safety of RTS,S/ASO1.

In Kenya, Olotu and colleagues followed children who had been enrolled in a small phase 2 RTS,S/AS01 trial for a period of seven years. ${ }^{5}$ These children had received three doses of RTS,S/AS01 when aged 5-17 months but not the booster dose. Vaccine efficacy (VE) against clinical episodes of malaria seen initially ${ }^{6}$ was lost during the later period of follow-up and in year five there was a negative efficacy of $-34.4 \%[95 \% \mathrm{Cl}-83.9,1.8]$ in the overall cohort and of $-56.8 \%[95 \% \mathrm{Cl}-118,7,-12.3]$ among children who lived in the area with the highest malaria transmission. ${ }^{5}$

In Lancet Infectious Diseases Tinto and colleagues report findings among children enrolled in three of the phase 3 trial centres who were followed for an additional three years. ${ }^{7}$ Encouragingly, VE against severe malaria was sustained; VE against severe malaria during the six or seven-year post vaccination period in children who had received a booster dose was $36.7 \%$ [95\% $\mathrm{Cl} 14.6,53.1]$ in the older age group and $31.0 \%[95 \% \mathrm{Cl} 4.7,50.0]$ in the younger age group. VE against uncomplicated malaria during the full follow-up period was less $23.7 \%$ [95\% $\mathrm{Cl} 15.9,30.7]$ and $15.5 \%$ [95\% Cl 6.7, 23.5] in the older and younger age groups respectively. No protection against clinical malaria was seen during the extension period and in the centre with the highest transmission, a statistically significant increase in the incidence of clinical malaria was seen in older children during the extension period (VE $30.3 \%[95 \% \mathrm{Cl}-59.5,-6.4])$. Fifteen deaths were recorded in RTS,S/AS01 vaccinated children (10 female, 5 male) and 7 in the controls ( 4 female, 3 male). Five cases of meningitis were reported, two in RTS,S/AS01 recipients and three in controls. Weaknesses of the study include a gap of nearly two years during which surveillance data was dependent on retrospective analysis of routinely collected data and the fact that only $70 \%$ of children in the original trial were enrolled in the extended follow-up. Nevertheless, this study provides strong evidence that RTS,S/AS01 can provide sustained protection against severe malaria 
and some reassurance on its safety, although numbers of serious adverse events and deaths were small and a gender imbalance was present in the latter.

In both studies, an increase in the incidence of uncomplicated clinical malaria was seen during the extended follow-up period, the effect being most marked in children exposed to the highest level of malaria transmission. This was not the case for severe malaria, although in Kenya, the peak age incidence of severe malaria was delayed in RTS,S/ASO1 vaccinated children. These findings are not surprising as there is strong evidence that repeated exposure to malaria is necessary to establish and sustain naturally acquired immunity although the intensity of infection needed to induce and sustain naturally acquired immunity is not known. However, some information comes from studies of chemoprevention. When malaria was prevented in Tanzanian infants during the first year of life a significant increase in cases of clinical malaria were seen in the following year. ${ }^{8}$ However, in children age 3-59 months protected for one year by seasonal malaria chemoprevention there was only a small increase in cases of uncomplicated malaria during the following year. ${ }^{9,10}$ Longer periods of protection may be followed by a substantial, although short-lasing, increase in susceptibility to clinical malaria ${ }^{11}$ but in all cases the benefits of protection exceeded those of the subsequent period of enhanced risk. It is likely that a highly effective malaria vaccine that provides only a relatively short period of protection will lead to some degree of 'rebound malaria' as its efficacy wanes unless the force of infection has been reduced during the period of follow-up. Booster vaccinations may delay the period of risk but vaccinated subjects may need to be provided with additional support during the period of enhanced risk through education, improved access to treatment and regular distribution of ITNs.

Words 732

\section{References}

1.RTS,S Clinical Trials Partnership. Efficacy and safety of RTS,S/AS01 malaria vaccine with or without a booster dose in infants and children in Africa: final results of a phase 3 , individually randomised, controlled trial. Lancet 2015; 386: 31-45.

2. Mendoza YG, Garric E, Leach A, et al. Safety profile of the RTS,S/AS01 malaria vaccine in infants and children: additional data from a phase III randomized controlled trial in sub- 
Saharan Africa. Hum Vaccin Immunother 2019 Apr 23:1-13. doi:

10.1080/21645515.2019.1586040. [Epub ahead of print].

3. WHO. Malaria vaccine: WHO position paper - January 2016. Wkly Epidemiol Rec 2016; 91: 33-52.

4. https://www.usnews.com/news/best-countries/articles/2019-04-30/test-malariavaccine-to-roll-out-in-ghana-and-kenya.

5. Bejon P, Lusingu J, Olotu A, et al. Efficacy of RTS,S/AS01E vaccine against malaria in children 5 to 17 months of age. N Engl J Med 2008; 359: 2521-32.

6. Olotu A, Fegan G, Wambua J, et al. Four-year efficacy of RTS,S/AS01E and its interaction with malaria exposure. N Engl J Med 2013; 368: 1111-20.

7. Tinto $\mathrm{H}$, Otieno W, Gesase $\mathrm{S}$, et al. Long-term incidence of severe malaria following RTS,S/AS01 vaccination in children and infants in Africa: an open three-year extension of a phase III randomized controlled trial. Lancet Infect Dis 2019; in press.

8. Menendez C, Kahigwa E, Hirt R, et al. Randomised placebo-controlled trial of iron supplementation and malaria chemoprophylaxis for prevention of severe anaemia and malaria in Tanzanian infants. Lancet 1997; 350: 844-50.

9. Dicko A, Barry A, Dicko M, et al. Malaria morbidity in children in the year after they had received intermittent preventive treatment of malaria in Mali: a randomized trial. PLoS One 2011; 6: e23390.

10. Konaté AT, Yaro JB, Ouédraogo AZ, et al. Morbidity from malaria in children in the year after they had received intermittent preventive treatment of malaria: a randomized trial. PLoS One 2011: 6: e23391.

11. Greenwood BM, David PH, Otoo-Forbes LN. Mortality and morbidity from malaria after stopping malaria chemoprophylaxis. Trans R Soc Trop Med Hyg 1995; 89: 629-33. 
9. 